\title{
Tratamiento médico exitoso de neumonía necrotizante en un paciente pediátrico
}

\author{
Herlina Uinarni $^{a-c}$ Felicia Nike $^{a} \quad$ Andi Dwi Bahagiad \\ a Departamento de Anatomía, Escuela de Medicina y Ciencias de la Salud, Universidad Católica Atma Jaya de Indonesia, Yakarta, Indonesia;

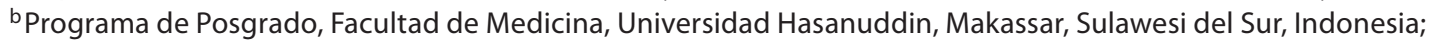 \\ 'Departamento de Radiología, Hospital de Maternidad y Pediatría Gran Familia, Yakarta, Indonesia; \\ ${ }^{\text {d} D e p a r t a m e n t o ~ d e ~ P e d i a t r i ́ a, ~ F a c u l t a d ~ d e ~ M e d i c i n a, ~ U n i v e r s i d a d ~ H a s a n u d d i n, ~ M a k a s s a r, ~ S u l a w e s i ~ d e l ~ S u r, ~ I n d o n e s i a ~}$
}

\section{Palabras clave}

Neumonía necrotizante $\cdot$ Niños · Estudios de imagen · Radiografía

torácica · TC torácica · Ultrasonografía pulmonar

\section{Resumen}

La neumonía necrotizante es una complicación grave pero poco frecuente de la neumonía en niños. Presentamos el caso de una niña de 20 meses de edad con dificultad respiratoria, que más adelante se diagnosticó como neumonía necrotizante. En este artículo

subrayamos el papel de estudios de imagen como la radiografía torácica, la TC torácica y la ultrasonografía pulmonar para el diagnóstico, y la importancia de la terapia intravenosa con antibióticos para un mejor resultado.

\section{Introducción}

La neumonía necrotizante (NN) es una complicación grave de la neumonía, caracterizada por la desmejora progresiva en niños previamente saludables, a pesar de la administración de antibióticos. Los patógenos más comúnmente asociados con la NN son neumococos y Staphylococcus aureus [1]. El manejo de pacientes con $\mathrm{NN}$ es complicado, porque no hay lineamientos firmes que describan el manejo de la NN. El pilar del tratamiento es el cuidado de soporte, con antibióticos apropiados. La intervención quirúrgica puede ser de utilidad cuando la terapia médica falla [2].

\section{Reporte de caso}

Una niña de 20 meses de edad se presentó en el Departamento de Emergencias (DE) con dificultad respiratoria progresiva y fiebre desde un día antes del ingreso. Su peso era $14 \mathrm{~kg}$, su temperatura corporal era $40^{\circ} \mathrm{C}$, y mostraba respiración rápida, sin estridor ni cianosis. Cinco días antes la había tratado el médico general, manifestando fiebre, tos y dificultad para respirar, y había recibido fármacos antipiréticos y antivirales, sin respuesta significativa. Su madre negó el antecedente de convulsiones febriles, afirmando que previamente había sido saludable y no tenía historial previo de padecimientos significativos. Nunca había recibido la vacuna contra Streptococcus pneumoniae ni Haemophilus influenzae tipo b (Hib).

Se obtuvo una muestra de sangre para análisis mientras la paciente se encontraba en el DE, incluyendo un recuento celular completo y electrolitos básicos. Los valores de laboratorio mostraron un conteo total de glóbulos blancos de $10,100 \mu \mathrm{L}^{-1}$, con $50 \%$ de neutrófilos segmentados. Su nivel de hemoglobina fue $10.2 \mathrm{~g} / \mathrm{dL}$, el conteo de plaquetas fue $470,000 \mu \mathrm{L}^{-1}$ y el nivel de proteína C-reactiva (CRP) fue $24 \mathrm{mg} / \mathrm{L}$. Los demás valores de laboratorio fueron poco notables.

La vista anteroposterior (AP) de la radiografía torácica obtenida por el departamento de radiología mostró neumonía lobar inferior izquierda (Figura 1). Luego de permanecer hospitalizada por cinco días, se observó una significativa mejoría clínica y en los parámetros de laboratorio, por lo que los padres solicitaron el alta voluntaria. 


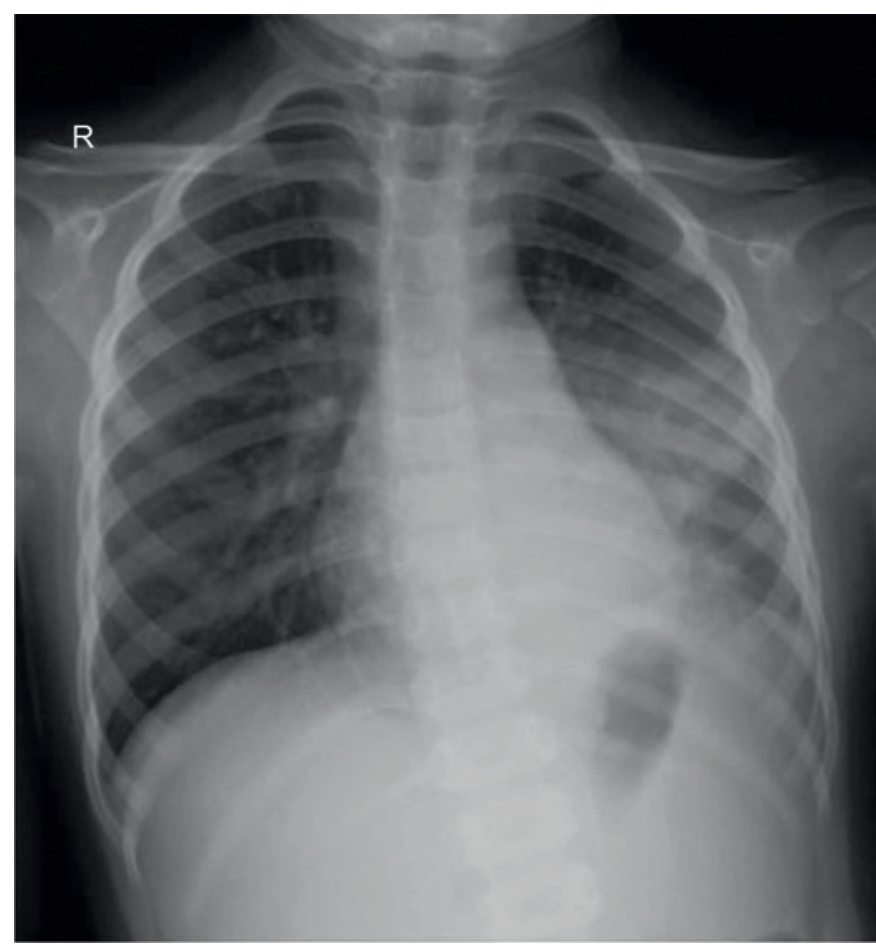

Fig. 1. Radiografía torácica AP-supina: consolidación visible en el lóbulo pulmonar inferior derecho. El pulmón derecho no mostró anormalidad aparente.
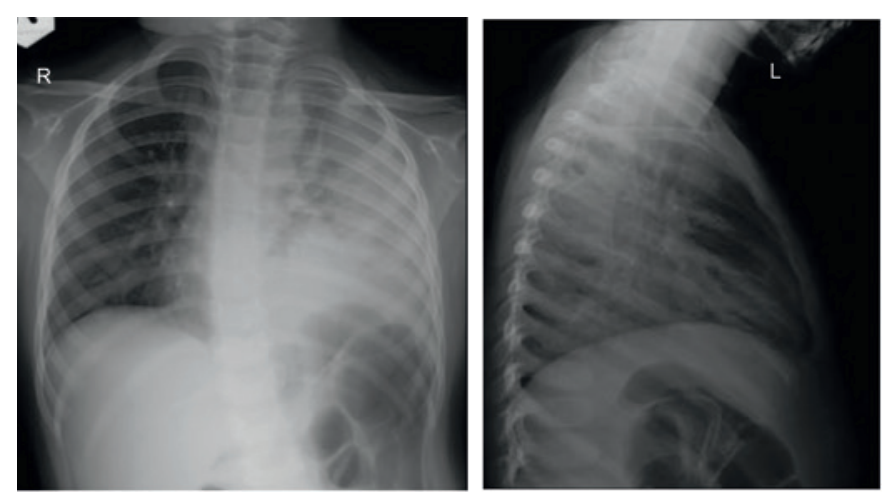

Fig. 2. Rx torácica con vista AP y lateral, que muestra un área focal de consolidación dominante en el lóbulo inferior izquierdo. En la vista AP hay múltiples lucencias pequeñas en el campo pulmonar izquierdo, sospechosas de una posible malformación congénita de las vías respiratorias (CPAM) y una posible neumonía con cavitación aérea. Puede compararse con la Rx torácica previa (Figura 1), donde no se observó lucencia alguna.

En su primer día en casa, los síntomas de fiebre y dificultad respiratoria empeoraron. Los padres llevaron a la paciente de regreso al DE. Tras su llegada al DE, su temperatura corporal era $38.7^{\circ} \mathrm{C}$, la frecuencia respiratoria era 52 veces por minuto, y la frecuencia cardiaca era 180 latidos por minuto. La paciente ingresó de nuevo al hospital, y las pruebas de laboratorio mostraron niveles elevados de CRP, $96 \mathrm{mg} / \mathrm{L}$, y un conteo alto de glóbulos blancos, 21,400 $\mu \mathrm{L}^{-1}$. La Tabla 1 muestra una comparación de los resultados de laboratorio. Se obtuvo una segunda Rx torácica en el departamento de radiologia (Figura 2). La vista AP y la lateral
Tabla 1. Comparación de valores de laboratorio entre el primer y el segundo periodo de hospitalización

\begin{tabular}{lrr}
\hline Parámetro (valores normales) & $\begin{array}{c}1^{\text {a }} \text { hospitali- } \\
\text { zación }\end{array}$ & $\begin{array}{l}2^{\text {a }} \text { hospitali- } \\
\text { zación }\end{array}$ \\
\hline Hemoglobina $\left(10.8-12.8 \mathrm{~g} \cdot \mathrm{dL}^{-1}\right)$ & 10.2 & 11.6 \\
Recuento leucocitos $\left(5,000-21,000 \mu \mathrm{L}^{-1}\right)$ & 10,100 & 21,400 \\
Recuento plaquetas $\left(150,000-450,000 \mathrm{\mu L}^{-1}\right)$ & 420,000 & 702,000 \\
Hematocrito (35-43\%) & 32.0 & 37.0 \\
\hline Conteo diferencial & & \\
Basófilos & 0 & 0 \\
Eosinófilos & 0 & 0 \\
Neutrófilos en banda & 4.0 & 1.0 \\
Neutrófilos segmentados & 50.0 & 81.0 \\
Linfocitos & 45.0 & 17.0 \\
Monocitos & 1.0 & 1.3 \\
Proteína C-reactiva $\left(<2 \mathrm{mg} \cdot \mathrm{L}^{-1}\right)$ & 24 & 96 \\
\hline
\end{tabular}

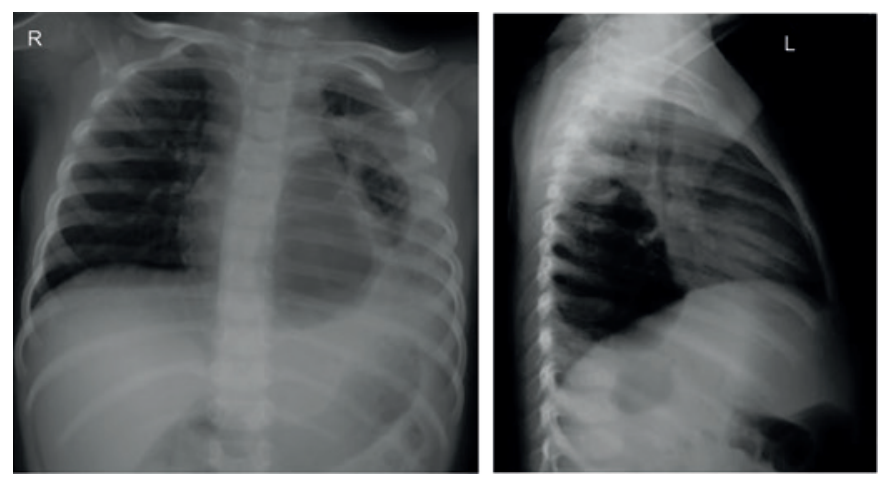

Fig. 3. Rx torácica en vista AP y lateral, que muestra una lesión cavitaria llena de aire en el lóbulo inferoposterior izquierdo.

demostraron un área focal de consolidación dominante en el lóbulo inferior izquierdo. En la vista frontal hay múltiples lucencias pequeñas en el campo pulmonar izquierdo, sospechosas de una posible neumonía con cavitación aérea, o de combinación con una malformación congénita de las vías respiratorias pulmonares (CPAM). Considerando que en la radiografía torácica previa ( $\mathrm{Fi}$ gura 1) no se observó lucencia alguna, es posible que se trate de neumonía con cavitación aérea. Durante la hospitalización, la condición de la paciente empeoró, y en el día 8 se tomó una $\mathrm{Rx}$ torácica de seguimiento (Figura 3) combinada con ultrasonografía pulmonar (USP) (Figuras 4 y 5), las cuales mostraron múltiples lesiones cavitarias llenas de aire en el pulmón izquierdo. Se observó efusión pleural (trasudativa), engrosamiento pleural y textura de eco parenquimal heterogénea, sin neumotórax. En el mismo día se practicó una TC torácica de exploración sin contraste, puesto que la paciente se resistió a la administración del contraste (Figuras 5-7). Las imágenes de TC de exploración revelaron una consolidación con múltiples cavidades o neumatoceles, consistentes con neumonía necrotizante, una densidad de efusión pleural izquierda $<25$ unidades Hounsfield (UH) y el colapso parcial del lóbulo superior izquierdo. El cultivo sanguíneo fue estéril, el aná- 

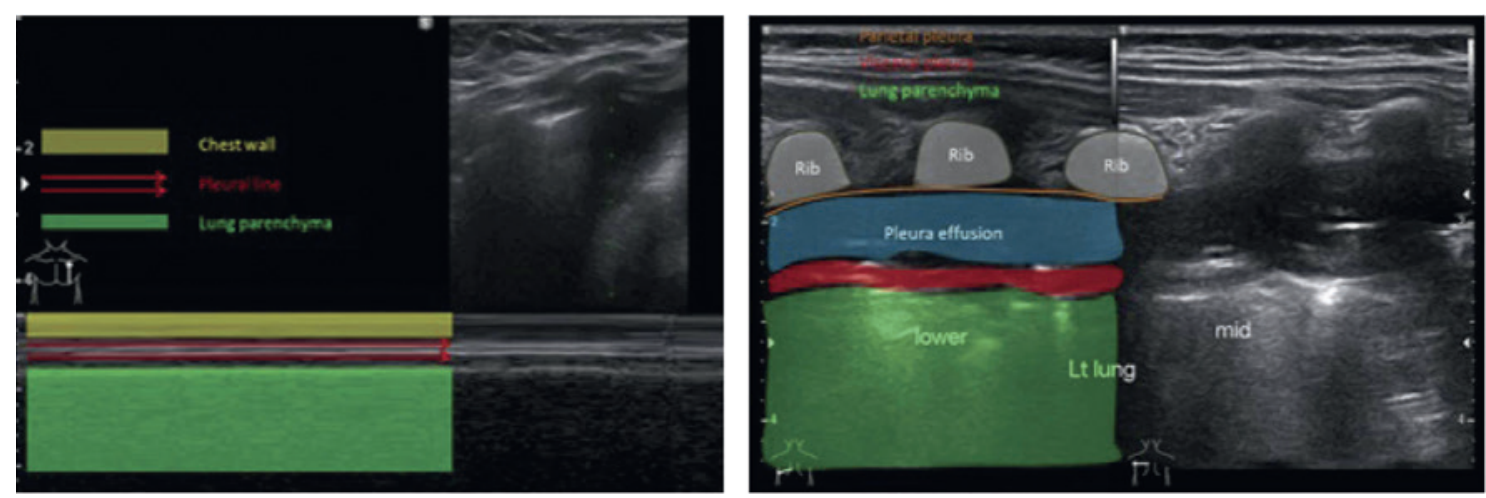

Fig. 4. La USP anterior izquierda, utilizando el modo M, no muestra neumotórax, y la posterior inferior y media, utilizando el modo B: efusión pleural (trasudado), engrosamiento pleural irregular y textura en eco parenquimal heterogénea.
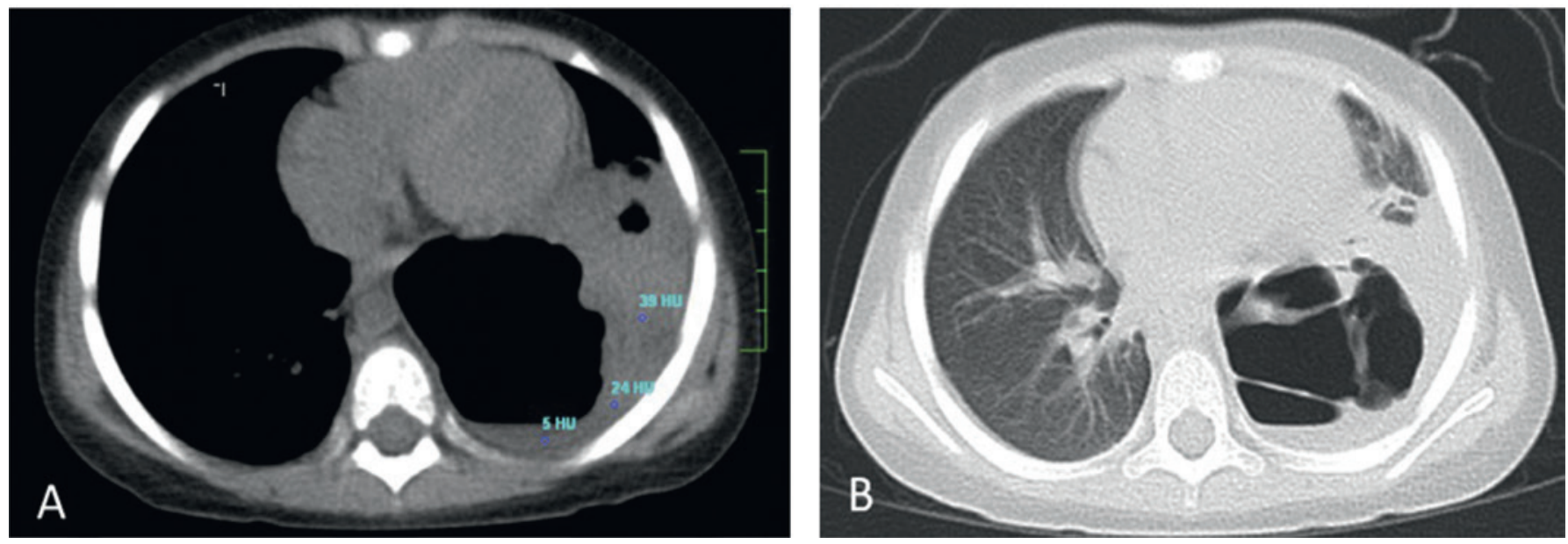

Fig. 5. TC axial-torácica sin contraste: ventana mediastinal (a) y ventana pulmonar (b). La densidad de la efusión pleural izquierda fue $<25$ UH. Hubo una porción poco visible del pulmón izquierdo, y una cavidad de pared fina llena de aire. Sin contraste IV, no pudo diagnosticarse necrosis cavitaria porque no hubo realce del entorno.
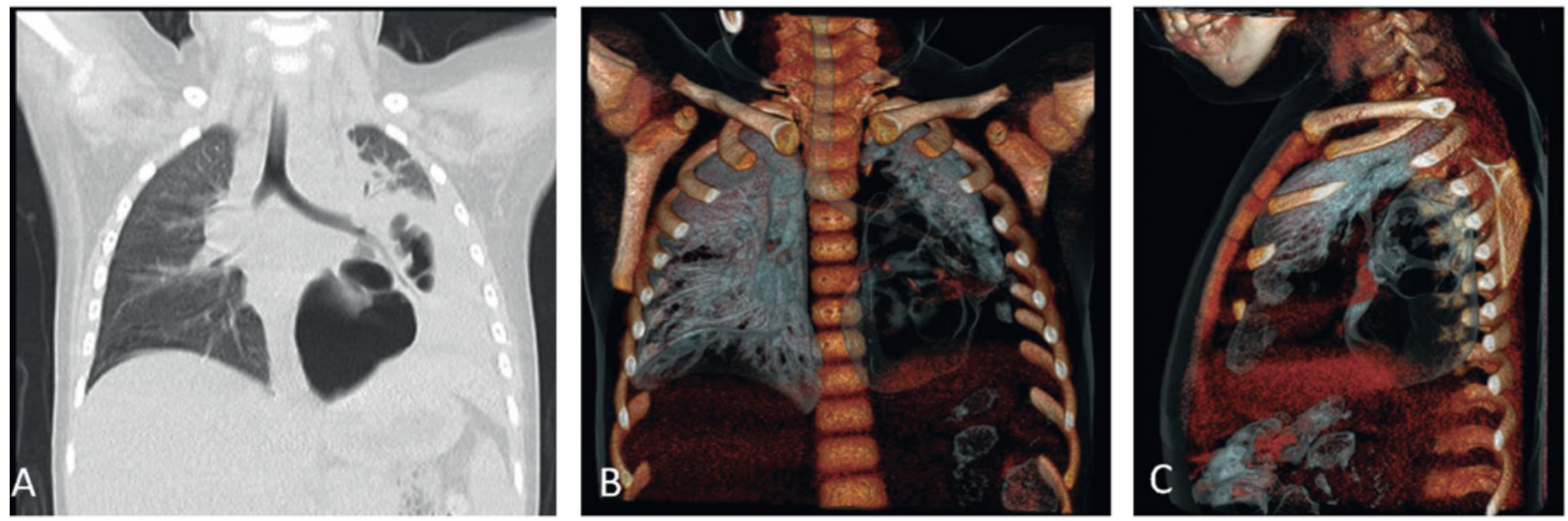

Fig. 6. TC torácica sin contraste, vista frontal: ventana pulmonar (a) y broncografía virtual (presentación con reconstrucción de volumen) para visualizar las vías respiratorias $(\mathbf{b}, \mathbf{c})$.

lisis citológico no reveló células tumorales malignas, y la tinción para bacilos acidorresistentes y la prueba GeneXpert también mostraron resultados negativos. La condición de la paciente mejoró con la administración intensiva de antibiótico por vía intra- venosa, consistente en meropenem, amikacina y azitromicina. En el tercer día de este periodo de hospitalización, la paciente mostró mejoría clínica significativa, incluyendo la resolución de la fiebre y la dificultad respiratoria. Siete días después, se le dio de alta y se 

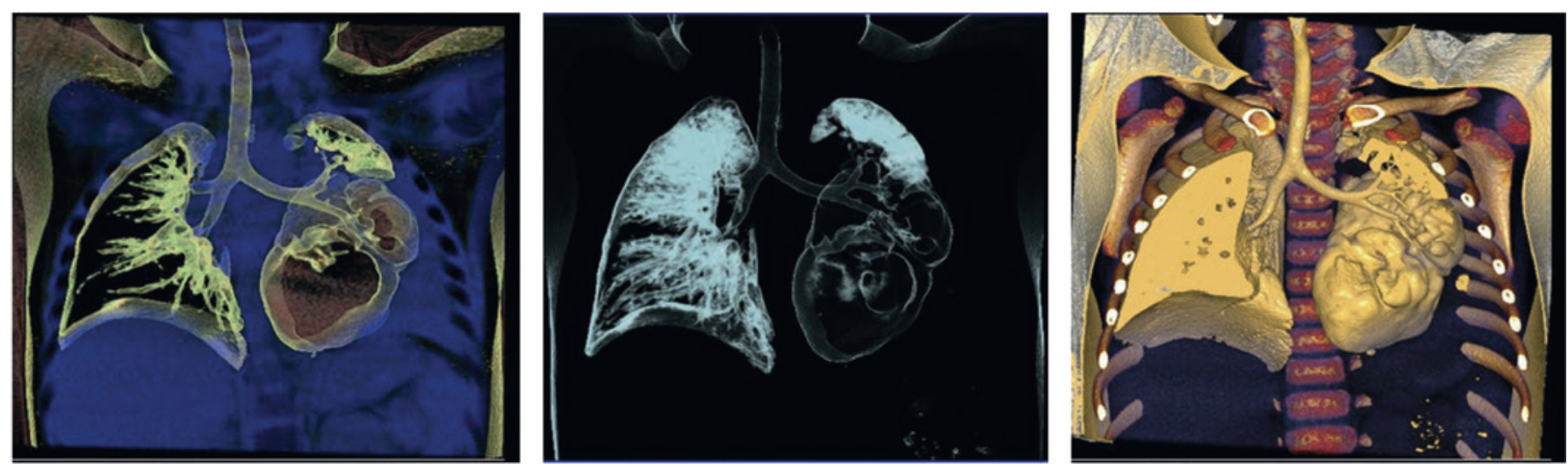

Fig. 7. TC torácica sin contraste, vista frontal, presentación con reconstrucción de volumen y reformateo de imagen: reformación coronal en ventana pulmonar estándar para permitir una visión tridimensional. Neumatoceles, desarrollo de cavidades con pared fina secundarias a necrosis.

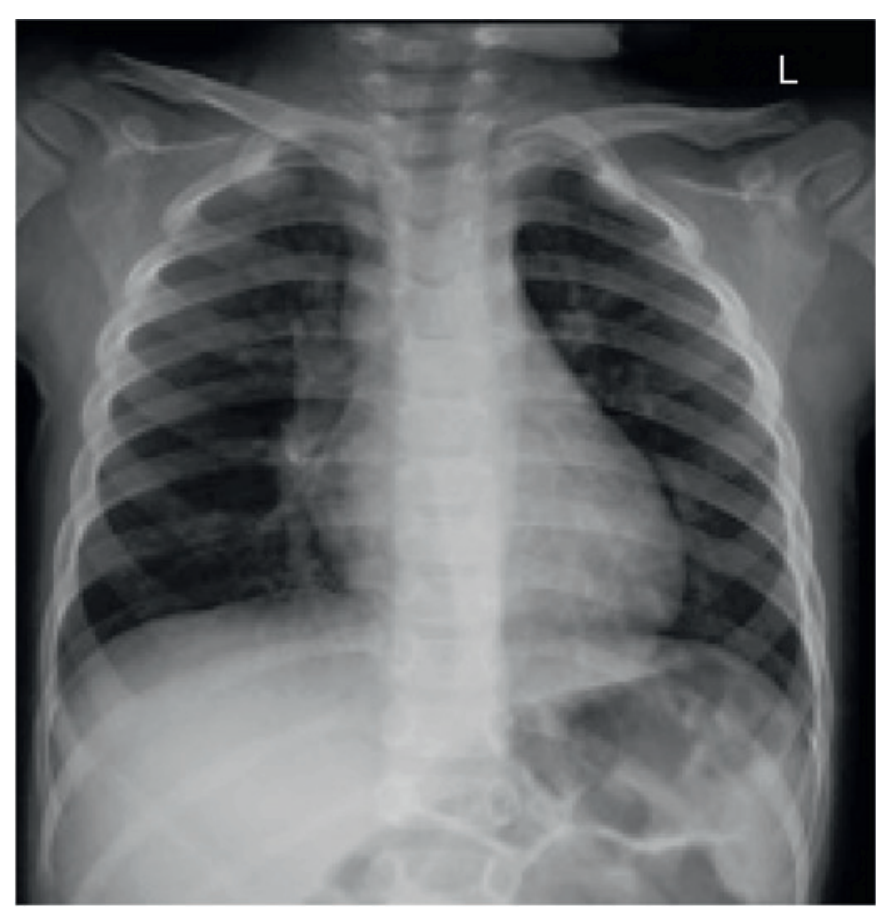

Fig. 8. Radiografía torácica de seguimiento obtenida seis meses después de la hospitalización. Rx torácica frontal sin anormalidades aparentes.

aconsejó continuar la terapia con antibióticos orales. Se sentía bien y volvió a sus actividades normales sin síntoma respiratorio alguno. Una radiografía torácica de seguimiento obtenida 6 meses después de la hospitalización no reveló anomalías (Figura 8).

\section{Discusión}

El término «neumonía necrotizante» $(\mathrm{NN})$ se refiere al desarrollo de necrosis, licuefacción y cavitación del parénquima pulmonar por patógenos infecciosos. Se dice que aproximadamente $4 \%$ de las neumonías comunitarias son necrotizantes, aunque este porcentaje parece incrementarse [3]. La falta de suministro vascular a los pulmones y los bronquios puede producir desvitalización del parénquima pulmonar. La falta de riego sanguíneo a las áreas subperfundidas puede causar una distribución reducida de antibióticos, permitiendo la progresión incontrolada de la infección y mayor destrucción del tejido pulmonar [4].

Usualmente, la NN se desarrolla por varios días, y se presenta de forma aguda con sepsis grave. Se encontraron un conteo bajo de leucocitos, tos sanguinolenta e hiperpirexia, junto con evidencia imagenológica de necrosis del tejido y microabscesos. Con frecuencia se observa gangrena pulmonar, y se forman grandes cavidades cuando los abscesos menores se unen. En contraste con la $\mathrm{NN}$, los abscesos pulmonares tienden a aparecer crónicamente, con una historia prolongada de fiebre y sudoraciones nocturnas [5]. En este caso, el niño desarrolla síntomas agudos, que ocurren en pocos días.

La radiología tiene un papel fundamental en el diagnóstico y el tratamiento de las complicaciones de la neumonía en niños. El estudio de imagen de elección es la radiografía torácica, incluso para la neumonía adquirida en la comunidad (NAC). La tomografía computada (TC), por otro lado, no es una herramienta de imagen de primera línea para niños con sospecha de NAC sin complicaciones. Se considera su uso cuando se sospecha que existan complicaciones o cuando es difícil diferenciar entre NAC y otras enfermedades [6]. La ultrasonografía pulmonar (USP) es otra modalidad de elección con una técnica de radiación no-ionizante asequible y portátil, que se ha utilizado para ayudar en el diagnóstico de patologías pulmonares en niños. Comparada con los rayos $\mathrm{X}$ y la TC, la USP tiene mayor sensibilidad y especificidad en el diagnóstico de NAC en pacientes pediátricos [7].

Con frecuencia, para tratar casos de NN se requiere un equipo multidisciplinario de médicos respiratorios pediátricos, cirujanos pediátricos o torácicos y expertos en enfermedades infecciosas. Los objetivos son controlar y en última instancia revertir los cambios patobiológicos asociados con la NN. Las medidas empleadas incluyen proporcionar oxígeno suplementario para aliviar la hipoxia, un manejo adecuado del dolor para reducir el dolor pleurítico causado por la infección y mejorar la ventilación, adminis- 
trar un ciclo prolongado de terapia con antibióticos y reducir cualquier efecto de masa o presión intratorácica al drenar el gas o el fluido intrapleural [8]. El tratamiento conservador se basa en la administración intravenosa (IV) de antibióticos. La terapia de primera línea recomendada para NAC grave sin complicación en niños consiste en penicilina o ampicilina IV, y después se requerirá incluir antibióticos antiestafilocócicos, como oxacilina o flucloxacilina. Cuando se sospecha de coinfección con Mycoplasma pneumoniae, se agrega un macrólido como claritromicina o azitromicina IV [1]. Además de la terapia conservadora, algunos autores recomiendan realizar procedimientos invasivos solamente si el niño sigue enfermo de forma grave y recurrente, a pesar de la terapia apropiada con antibióticos [9]. En este caso, administramos terapia conservadora a la paciente, con una administración intensiva de antibióticos intravenosos, entre ellos meropenem, amikacina y azitromicina, y el resultado fue notable.

\section{Conclusión}

La neumonía necrotizante representa una complicación rara, pero grave, de la neumonía en niños. Debe sospecharse de ella en casos de neumonía con deterioro clínico progresivo, fiebre alta y dificultad respiratoria a pesar de una terapia adecuada con antibióticos, por al menos 72 horas. La USP y la TC son modalidades imagenológicas bien establecidas para la evaluación de neumonía pediátrica complicada. No debe retrasarse el uso de la TC exploratoria para confirmar el diagnóstico y proporcionar tratamiento. Inicialmente, debe administrarse tratamiento conservador con oxígeno suplementario y antibióticos IV, mientras que debe brindarse tratamiento quirúrgico e invasivo si el niño sigue gravemente enfermo y la infección es refractaria.

\section{Conflictos de interés}

Los autores declaran que no tienen conflictos de interés.

\section{Información de licencias}

(ㅇ 2020 Herlina Uinarni, Felicia Nike, Andi Dwi Bahagia: A Successful Medical Treatment of Necrotizing Pneumonia in a Pediatric Patient. Case Rep Pediatr. 2020; 2020: 8875119. (traducción), con licencia bajo CC BY 4.0 (https://creativecommons.org/licenses/by/4.0/deed.es).

\section{Referencias}

Las referencias están disponibles en www.karger.com/Article/Fulltext/ 000516173 . 